\title{
Rapid Triage of Mental Health Risk in Emergency Medical Workers: Findings From Typhoon Haiyan - CORRIGENDUM
}

Lauren Sylwanowicz; Merritt Schreiber; Craig Anderson; Carlos Primero D. Gundran; Emelie Santamaria; Jaifred Christian F. Lopez; Hilton Lam; Anna Cristina Tuazon

doi: 10.1017/dmp.2017.37, Published by Cambridge University Press, 14 September 2017.

$\mathrm{T}$

he original publication of this article omitted two of the article authors. The authors apologize for this error. The article has since been corrected.

\section{REFERENCE}

Sylwanowicz L, Schreiber M, Anderson C, Gundran CPD, Santamaria E, Lopez JCF, Lam H, Tuazon AC. Rapid Triage of Mental Health Risk in Emergency Medical Workers: Findings From Typhoon Haiyan. Disaster Med Public Health Preparedness. 2018;12:19-22. doi: 10.1017/dmp.2017.37. 